\title{
To know any disease well is to have knowledge and awareness of the possibility of overlapping sleep disorders
}

\section{Kazuo Chin ${ }^{1}$}

Published online: 4 October 2019

C Japanese Society of Sleep Research 2019
The prevalence of sleep disorders is very high. Therefore, sleep disorders are very likely to occur in the presence of other diseases. Some of these conditions interact with sleep disorders. Sleep related breathing disorders (SRBD) are among the major sleep disorders [1]. Obstructive sleep apnea (OSA) has the highest prevalence, among SRBD. When a patient with chronic obstructive pulmonary disease (COPD) develops OSA, it is called overlap syndrome [2]. It was reported that patients with overlap syndrome have a poorer prognosis than patients with COPD only [3]. Therefore, it is important to determine whether or not a patient has SRBD in addition to the original disease. Regarding this issue, Saçmaci et al. [4] reported on sleep disorders, including SRBD in patients with multiple sclerosis (MS). They investigated the relationship between the demyelinating plaque lesion load associated with brain localization and sleep breathing values and structure of sleep in patients with relapsing-remitting MS (RRMS) who underwent polysomnography. They found a negative correlation between the percentage of REM (\%) and infratentorial and brainstem lesion loads (rho: -0.396 , $-0.418 ; p<0.05$ ) by brain magnetic resonance imaging (MRI) in 28 patients with MS. Sleep efficiency was adversely affected due to supratentorial lesion loads, but without statistical significance $(p=0.06)$. There was no statistically significant correlation between total brain lesion loads and sleep breathing parameters, including OSA and the desaturation index $(p>0.05)$. Usually, it is said that patients with MS have SRBD. But in this study, the oxygen desaturation index, obstructive apnea, and the apnea-hypopnea index were not significantly associated with the total lesion load or the infratentorial lesion load by MRI. Previously in this journal, patients with OSA had serum substance P (SP) levels lower

Kazuo Chin

chink@kuhp.kyoto-u.ac.jp

1 Department of Respiratory Care and Sleep Control Medicine, Graduate School of Medicine, Kyoto University, Kyoto, Japan than snorers and there was a significant positive correlation between serum levels of SP and the time spent in REM sleep (\% of total sleep time) [5]. Following 3 months of continuous positive airway pressure treatment, the serum SP levels in patients with OSA significantly decreased. Lesions determined by MRI in patients with MS and SP levels in OSA patients were significantly correlated with the percentage of REM (\%). Thus, changes in brain lesions and a humoral factor (SP) might have some effects on sleep structure. From these studies, we are able to recognize that sleep disorders such as SRBD itself or the effects of several diseases may influence changes in sleep structures and that changes in sleep structures may have several effects on the course of diseases and in turn mortality.

Thus, at present, it is important for physicians to be aware of overlapping sleep disorders, if they want to know their patients well and control their diseases, which may have the possibility of being accompanied by sleep disorders.

\section{References}

1. International classification of sleep disorders. Third edition. American Academy of Sleep Medicine. Dairen. 2014.

2. Flenley DC. Sleep in chronic obstructive lung disease. Clin Chest Med. 1985;6:51-61.

3. Marin JM, Soriano JB, Carrizo SJ, Boldova A, Celli BR. Outcomes in patients with chronic obstructive pulmonary disease and obstructive sleep apnea: the overlap syndrome. Am J Respir Crit Care Med. 2010;182(3):325-31.

4. Saçmaci H, Tanik N, Ökçesiz İ, İntepe YS, Aktürk T, Çiftçi B, İnan LV. The association of brain lesion locations and sleep parameters in patients with multiple sclerosis: a pilot study. Sleep Biol Rhythms. 2019. https://doi.org/10.1007/s41105-019-00231-2.

5. Guclu OA, Ursavas A, Kasapoglu F, Ozarda Y, Bozyigit C, Ocahoglu G, Karadag M. Relationship with excessive daytime sleepiness and serum substance $P$ levels in OSAS patients and the effect of PAP treatment. Sleep Biol Rhythms. 2019;17:355-61.

Publisher's Note Springer Nature remains neutral with regard to jurisdictional claims in published maps and institutional affiliations. 